\title{
A Study of 100 Cases of Cervical Schwannoma Treated with Inter-Capsular Resection
}

\author{
Toshikazu Shimane ${ }^{1,2,3 *}$, Shunya Egawa ${ }^{1,2,3}$, Yukiomi Kushihashi ${ }^{1,2,3}$, Hitoshi Sato ${ }^{1,3}$, \\ Yoshiro Saito $^{1,3}$, Yuya Kurasawa ${ }^{1,3}$, Takashi Moriya ${ }^{1,3}$, Hirano Koujiro², Hideyuki Katsuta1,2,3, \\ Kenichiro Ikeda1,2,3 \\ ${ }^{1}$ Showa University Head and Neck Oncology Center, Tokyo, Japan \\ ${ }^{2}$ Department of Otorhinolaryngology, Showa University, School of Medicine, Tokyo, Japan \\ ${ }^{3}$ Department of Oral and Maxillofacial Surgery, Division of Oral Oncology, Showa University, School of Dentistry, Tokyo, Japan \\ Email: *shima-tskz@dent.showa-u.ac.jp
}

How to cite this paper: Shimane, T., Egawa, S., Kushihashi, Y., Sato, H., Saito, Y., Kurasawa, Y., Moriya, T., Koujiro, H., Katsuta, H. and Ikeda, K. (2020) A Study of 100 Cases of Cervical Schwannoma Treated with Inter-Capsular Resection. International Journal of Otolaryngology and Head \& Neck Surgery, 9, 61-67.

https://doi.org/10.4236/ijohns.2020.92009

Received: January 21, 2020

Accepted: February 24, 2020

Published: February 27, 2020

Copyright $\odot 2020$ by author(s) and Scientific Research Publishing Inc. This work is licensed under the Creative Commons Attribution International License (CC BY 4.0).

http://creativecommons.org/licenses/by/4.0/ (c) (i) Open Access

\begin{abstract}
Background: Cervical schwannoma is a relatively rare disease, and it is difficult to experience many surgical cases because it may be followed up without surgery. We examined 100 patients who underwent inter-capsular resection for cervical schwannomas at our center and classified the patients according to the nerve of origin. Methods: We retrospectively reviewed 100 patients who underwent inter-capsular resection for cervical schwannoma at our center from April 2005 to September 2019. We examined the patient's characteristics including age, sex, tumor size (maximum diameter), origin nerve, preoperative symptoms, and postoperative neurological deficits for all cases. We classified the cases according to the nerve of origin and the occurrence of postoperative neurological deficits. Results: The occurrence of postoperative neurological deficit for all cases was as follows: "none" was 73\%, "temporary paralysis" was $21 \%$, and "permanent paralysis" was $6 \%$. In the case of vagus nerve: "none" was $65.4 \%$, "temporary paralysis" was $23.1 \%$, "permanent paralysis" was $11.5 \%$. In the case of sympathetic nerve: "none" was $64.7 \%$, "temporary paralysis" was $29.4 \%$, "permanent paralysis" was $5.9 \%$. In the case of brachial plexus: "none" was $87.0 \%$, "temporary paralysis" was $13.0 \%$, "permanent paralysis" was $0 \%$. In the case of cervical and accessory nerves: "none" was $86.4 \%$, "temporary paralysis" was $13.6 \%$, "permanent paralysis" was $0 \%$. In the case of facial nerve: "none" was $0 \%$, "temporary paralysis" was $80.0 \%$, "permanent paralysis" was $20 \%$. In the case of lingual nerve: "none" was $80.0 \%$, "temporary paralysis" was $20.0 \%$, "permanent paralysis" was $0 \%$. Conclusions: Inter-capsular resection is useful for the treatment of cervical schwannoma and a simple comparison is difficult, but probably with good
\end{abstract}


results. This study provides information that will be useful for the treatment of cervical schwannoma.

\section{Keywords}

Cervical Schwannoma, Inter-Capsular Resection, Origin Nerve, Neurological Deficit, Complication

\section{Introduction}

Cervical schwannoma is a relatively rare disease [1] [2] [3], and it is difficult to experience many surgical cases because it may be followed up without surgery. In addition, there are few reports that examined the incidence of postoperative neurological deficits, and there are no reports that examined postoperative neurological deficits according to the nerve of origin. In our center, the same surgeon actively performs cervical schwannoma surgery using the same method and has treated several patients with cervical schwannoma. In this study, we retrospectively reviewed 100 patients who underwent inter-capsular resection for cervical schwannoma at our center and classified these patients according to the nerve of origin. This information will be beneficial for the treatment of cervical schwannomas in the future.

\section{Methods}

We retrospectively reviewed 100 patients who underwent inter-capsular resection for cervical schwannoma at our center from April 2005 to September 2019. 100 patients are all cases during this period. We examined the patient's characteristics including age, sex, tumor size (maximum diameter), origin nerve, preoperative symptoms, and postoperative neurological deficits for all cases. In addition, these cases were classified according to the nerve of origin for tumors with at least 5 cases. The accessory nerve and the cervical nerve were examined collectively. We evaluated the postoperative neurological deficits as follows: "none" showed no new symptoms, "temporary paralysis" improved within 6 months after surgery, and "permanent paralysis" remained after 6 months.

\section{Results}

\subsection{Overall Characteristics $(n=100)$}

The mean age of the patients was 46.4 years (range, $16-71$ years). The ratio of male to female patients was 53:47. The mean tumor diameter was $40.9 \mathrm{~mm}$ (range, $15-84 \mathrm{~mm}$ ). There were $26,23,17,19,5,5,3,1$, and 1 cases of vagus nerve, brachial plexus, sympathetic nerve, cervical nerve, facial nerve (parotid gland), lingual nerve, accessory nerve, superior laryngeal nerve, and recurrent laryngeal nerve, respectively (Figure 1). Preoperative symptoms were observed 


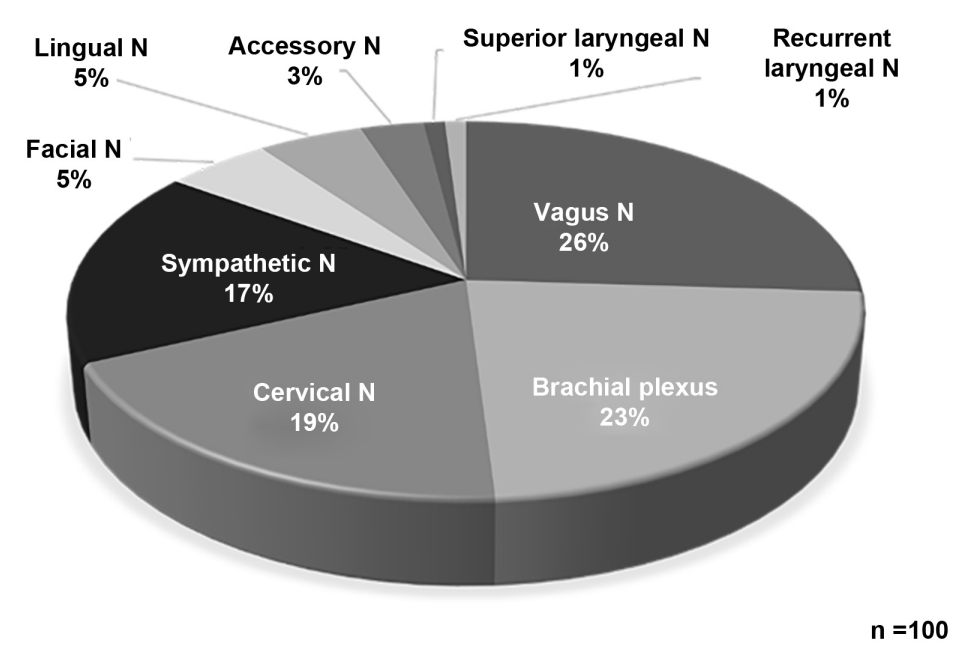

Figure 1. Origin nerves of schwannoma.

in $21 \%$ of the cases, while $79 \%$ of the cases reported no preoperative symptoms. Postoperative neurological deficit was not observed in $73 \%$ of the patients, whereas $21 \%$ and $6 \%$ of the patients had temporary and permanent paralysis, respectively (Figure 2).

\subsection{Classification According to the Nerve of Origin}

The cases were classified according to the nerve of origin are shown in Figure 3.

In the case of the vagus nerve, the mean age of the patients was 44.1 years (range, 21 - 71 years). The ratio of male to female patients was 11:15. The mean tumor diameter was $46.6 \mathrm{~mm}$ (range, 20 - $84 \mathrm{~mm}$ ). Preoperative symptoms (cough) were observed in $7.7 \%$ ( 2 cases) of the cases, while 92.3\% (24 cases) of the cases reported no preoperative symptoms. Postoperative neurological deficit (laryngeal palsy or cough) was not observed in $65.4 \%$ (17 cases) of the patients, whereas $23.1 \%$ (6 cases) and $11.5 \%$ ( 3 cases) of the patients had temporary and permanent paralysis, respectively.

In the case of the sympathetic nerve, the mean age of the patients was 44.6 years (range, $61-71$ years). The ratio of male to female patients was 6:11. The mean tumor diameter was $43 \mathrm{~mm}$ (range, $26-83 \mathrm{~mm}$ ). Preoperative symptoms (Horner's sign) were observed in $11.8 \%$ ( 2 cases) of the cases, while $88.2 \%$ (15 cases) of the cases reported no preoperative symptoms. Postoperative neurological deficit (Horner's sign) was not observed in 64.7\% (11 cases) of the patients, whereas $29.4 \%$ (5 cases) and $5.9 \%$ (1 case) of the patients had temporary and permanent paralysis, respectively.

In the case of the brachial plexus nerve, the mean age of the patients was 51.3 years (range, 30 - 66 years). The ratio of male to female patients was 16:7. The mean tumor diameter was $40.4 \mathrm{~mm}$ (range, 15 - $78 \mathrm{~mm}$ ). Preoperative symptoms (sensory and motor disorders) were observed in $47.8 \%$ (11 cases) of the cases, while $52.2 \%$ (12 cases) of the cases reported no preoperative symptoms. Postoperative neurological deficit (sensory and motor disorders) was not 


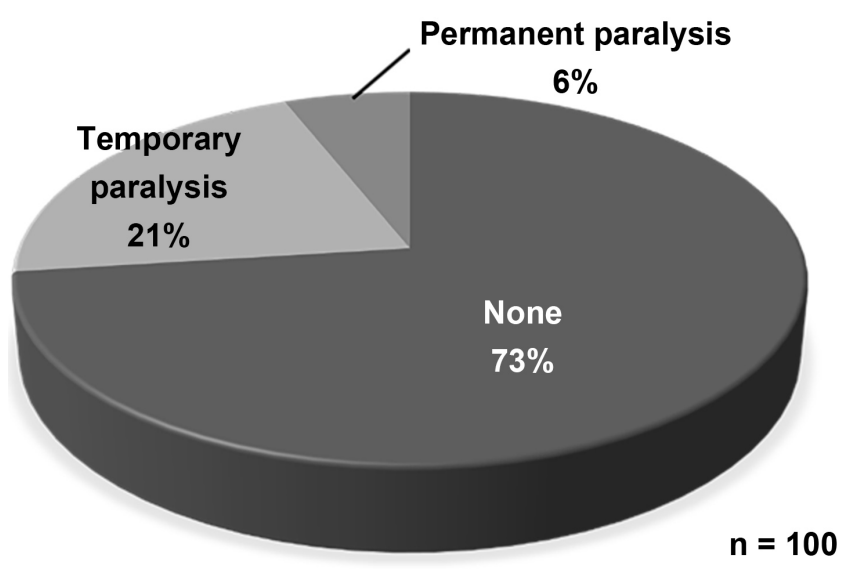

Figure 2. Postoperative neurological deficit.

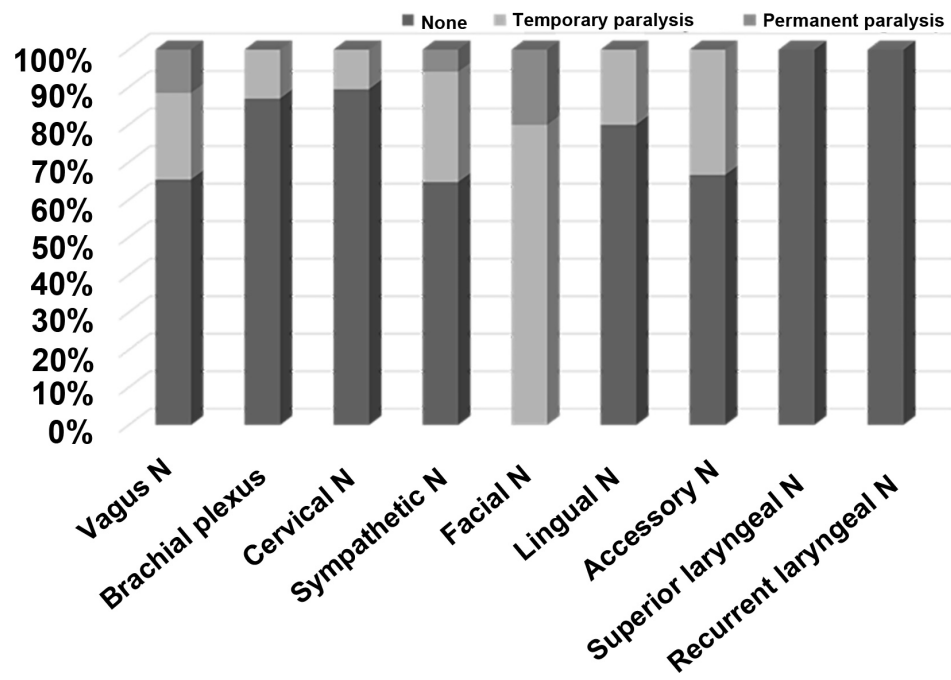

Figure 3. Postoperative neurological deficit (origin nerve classification).

observed in $87 \%$ ( 20 cases) of the patients, whereas $13 \%$ ( 3 cases) of the patients had temporary paralysis.

In the case of cervical and accessory nerves, the mean age of the patients was 45.9 years (range, 18 - 65 years). The ratio of male to female patients was 7:4. The mean tumor diameter was $30.5 \mathrm{~mm}$ (range, $18-67 \mathrm{~mm}$ ). Preoperative symptoms (sensory and motor disorders) were observed in $22.7 \%$ (5 cases) of the cases, while $77.3 \%$ (17 cases) of the cases reported no preoperative symptoms. Postoperative neurological deficit (sensory and motor disorders) was not observed in $86.4 \%$ ( 19 cases) of the patients, whereas $13.6 \%$ ( 3 cases) of the patients had temporary paralysis.

In the case of the facial nerve, the mean age of the patients was 44.2 years (range, 24 - 65 years). The ratio of male to female patients was 3:2. The mean tumor diameter was $44.7 \mathrm{~mm}$ (range, $22-73 \mathrm{~mm}$ ). Preoperative symptoms (facial paralysis) were observed in $20 \%$ ( 1 case) of the cases, while $80 \%$ ( 4 cases) of the cases reported no preoperative symptoms. Postoperative neurological deficit (facial paralysis) was observed $80 \%$ (4 cases) and 20\% (1 case) of the patients had 
temporary and permanent paralysis, respectively.

In the case of the lingual nerve, the mean age of the patients was 46 years (range, 16 - 67 years). The ratio of male to female patients was 1:4. The mean tumor diameter was $36.4 \mathrm{~mm}$ (range, 28 - $66 \mathrm{~mm}$ ). Preoperative symptoms were not observed in all cases. Postoperative neurological deficit (sensory disorders) was not observed in $80 \%$ ( 4 cases) of the patients whereas $20 \%$ ( 1 case) of the patients had temporary paralysis, respectively.

\section{Discussion}

Surgical methods for cervical schwannoma include total excision that cuts and removes the nerve at both ends of the tumor, subcapsular excision that cuts the tumor capsule and removes it inside the capsule, and inter-capsular resection that cuts the epineurium and separates the nerve from the true tumor capsule. This classification is frequently used in Japan; however, there are some situations where this classification is not clearly defined. Various names such as intracapsular enucleation [1] [2], intracapsular microenucleation [3], and intracapsular excision [4], are used and reported outside Japan, but there is no clear indication regarding which method these names correspond to. Inter-capsular resection is a technique devised by Hashimoto [5]. In this method, the tumor is removed while retaining the maximum number of normal nerve bundles. A surgeon cuts the epineurium on the tumor side with no normal nerve bundle and removes only the true tumor after cutting the perineurium. It is relatively difficult to identify and remove the true tumor capsule. Previously, we reported how Narrow Band Imaging (NBI) can be used to confirm the true tumor capsule [6]. Moreover, we devised a schwannoma model and a mechanism that could not be removed unless nerve fibers that do not need to be cut if they were detached at the wrong layer [7]. Considering the difference between enucleation and inter-capsular resection, incision of the epineurium and removal with either the wrong layer or the true tumor capsule is enucleation; on the other hand when the tumor is properly removed with a true tumor capsule, it is considered as an intracapsular resection. Therefore, we consider that enucleation belongs to a large category, and inter-capsular resection belongs to a small category.

Our center is consistently performing inter-capsular resection for cervical schwannoma since 2005. Since there were only a few cases per year initially, we could not significantly improve the surgical technique. Subsequently, the number of cases gradually increased, and the number of referral cases increased from 15 to 20 per year. Contrarily, several cases of relatively large tumors were followed up at other hospitals and often underwent difficulty surgeries.

Although cervical schwannoma may occur at any age, most cases are of 30 50 years of age, and we observed a similar trend in this study. Moreover, consistent with previous reports, no gender difference was observed in this study [8].

The size of the tumor varies in the $30-40 \mathrm{~mm}$ range [8]. In our study, several tumors were slightly larger than those reported previously. This may be because several cases were referred from other hospitals after the tumor increased in size. 
Previously, it was reported that the most common nerve of origin was the vagus nerve followed by the sympathetic nerve, the brachial plexus, and the cervical nerve [2]. We obtained similar results with $26 \%, 23 \%, 19 \%$, and $17 \%$ of the cases for vagus nerve, brachial plexus, cervical nerve, and sympathetic nerve, respectively.

The most common preoperative symptom is neck mass. According to previous reports, about $70 \%$ of cases have no preoperative symptoms [8]. In this study, $21 \%$ of cases had preoperative symptoms, and $79 \%$ of cases had only cervical mass and no other preoperative symptoms. According to the nerve of origin, in the vagus nerves, "cough" was observed in $7.7 \%$ cases ( 2 cases) and "none" in $92.3 \%$ cases ( 24 cases); in the brachial plexus, sensory and motor disorders were observed in $47.8 \%$ cases (11 cases) and "none" in 52.2\% cases (12 cases); in the cervical and accessory nerves, sensory and motor disorders were observed in $22.7 \%$ cases ( 5 cases) and "none" in $77.3 \%$ cases (17 cases); in the sympathetic nerve, Horner's sign was observed in $11.8 \%$ cases ( 2 cases) and "none" in $88.2 \%$ cases (15 cases); in the facial nerves, facial paralysis was observed in $20 \%$ cases ( 1 case) and "none" in $80 \%$ cases ( 4 cases). These results indicate that schwannoma derived from the brachial plexus frequently causes preoperative symptoms. In the brachial plexus, $56 \%$ of cases were reported to have preoperative symptoms [9].

There were reports of postoperative neurological deficits. However, since most of the reports vary with respect to the surgical methods, operators, and tabulation of reports, a simple comparison with previous studies is difficult. In addition, they describe a small number of cases, while this report describes a relatively large number of cases. Hirano et al. [10] reported that 11 of 21 (52.4\%) patients (total excision 11 cases, undercapsular enucleation9 cases, partial excision 1 case) had a postoperative neurological deficit in 22 years. Yasumatsu et al. [2] reported that 16 of 27 (59.3\%) patients (total excision 11 cases, intracapsular enucleation 16 cases) had a postoperative neurological deficit in 7 years. Kim et al. [1] reported that 2 of 7 (28.6\%) patients (intracapsular enucleation) had a postoperative neurological deficit in 6 years. In our study, $73 \%$ had none of the postoperative neurological deficits, $21 \%$ had temporary paralysis, and $6 \%$ had permanent paralysis. Inter-capsular resection is useful for the treatment of cervical schwannoma and a simple comparison is difficult, but probably with good results.

\section{Conclusion}

We retrospectively reviewed 100 cases of inter-capsular resection for cervical schwannoma performed at our center and classified them according to the nerve of origin. Inter-capsular resection is useful for the treatment of cervical schwannoma and a simple comparison is difficult, but probably with good results. This information will be beneficial to clinicians and surgeons for the treatment of cervical schwannomas in the future. 


\section{Conflicts of Interest}

The authors declare no conflicts of interest regarding the publication of this paper.

\section{References}

[1] Kim, S.H., Kim, N.H., Kim, K.R., Lee, J.H. and Choi, H.S. (2010) Schwannoma in Head and Neck: Preoperative Imaging Study and Intracapsular Enucleation for Functional Nerve Preservation. Yonsei Medical Journal, 51, 938-942. https://doi.org/10.3349/ymj.2010.51.6.938

[2] Yasumatsu, R., Nakashima, T., Miyazaki, R., Segawa, Y. and Komune, S. (2013) Diagnosis and Management of Extracranial Head and Neck Schwannomas: Review of 27 Cases. International Journal of Otolaryngology, Article ID: 973045, 5 p. https://doi.org/10.1155/2013/973045

[3] Rigante, M., Petrelli, L., De Corso, E. and Paludetti, G. (2015) Intracapsular Microenucleation Technique in Case of Intraparotid Facial Nerve Schwannoma. Technical Notes for a Conservative Approach. ACTA Otorhinolaryngologica Italica, 35, 49-52.

[4] Kang, G.C., Soo, K.C. and Lim, D.T. (2007) Extracranial Non-Vestibular Head and Neck Schwannomas: A Ten-Year Experience. Annals, Academy of Medicine, Singapore, 36, 228-233.

[5] Hashimoto, S. (2007) Concept of Inter-Capsular Resection for Cervical Schwannoma. Head \& Neck Surgery, 17, 91-92. https://doi.org/10.5106/jijshns.17.91

[6] Shimane, T., Ikeda, K., Kushibashi, Y., Egawa, S., Ikeya, Y., Kitajima, T., et al. (2017) Surgical Resection of Cervical Schwannoma with the Use of Narrow Band Imaging. Head \& Neck Surgery, 27, 211-215. https://doi.org/10.5106/jjshns.27.211

[7] Shimane, T., Kushibashi, Y., Egawa, S. and Ikeda, K. (2019) Reproduction of Intercapsular Resection for Cervical Schwannoma Using a Model. Nihon Kikan Shokudoka Gakkai Kaiho, 70, 363-368. https://doi.org/10.2468/jbes.70.363

[8] Kida, A., Fujii, M., Anazawa, U., Anasawa, U., Iida, H., Endo, S. and Yasuda, M. (1990) Report of Five Cases and Review of Japanese Literatures. Práctica en ORL, 83, 275-286. https://doi.org/10.5631/jibirin.83.275

[9] Shimane, T., Shimotatara, Y., Nakamura, T., Kawamura, Y., Ikenoya, Y, Takahashi G, et al. (2014) Examination of Cervical Neurinomas of the Brachial Plexus. Head \& Neck Surgery, 24, 189-193. https://doi.org/10.5106/jijshns.24.189

[10] Hirano, S., Kitamura, H., Miyata, K., Kaneko, K. and Asato, R. (1994) Extracranial Neurinomas of Head and Neck. Práctica en ORL, 87, 253-257. https://doi.org/10.5631/jibirin.87.253 